Discrete Comput Geom 34:463-474 (2005)

DOI: $10.1007 / \mathrm{s} 00454-005-1181-3$

\title{
New Upper Bound on the Transversal Width of T(3)-Families of Discs*
}

\author{
Aladár Heppes \\ Rényi Institute of the Hungarian Academy of Sciences, \\ H-1053 Budapest, Hungary \\ aheppes@renyi.hu
}

\begin{abstract}
A result of Eckhoff [3] implies that to every finite $T$ (3)-family of pairwise disjoint copies of a closed disc of unit diameter there exists a strip of width 1 meeting all members of the family. Our goal is to generalize this result giving a stricter upper bound by proving that the narrowest transversal strip has width $<0.65$.
\end{abstract}

\section{Introduction, Definitions, Results}

Throughout this paper the term disc is used for a closed solid circle of diameter 1 and unit disc means a closed solid circle of radius $1 . D(X)$ and $U(Y)$ denote the disc centered at $X$ and the unit disc centered at $Y$.

A line transversal to a family of discs is a straight line having a non-empty intersection with every member of the family. Alternatively, we also say that this family has property $T$. A family of at least $k$ discs is a $T(k)$-family if every $k$-member subset of it has property $T$.

Problems concerning line transversals of families of discs, especially Helly-type questions, have been studied by many authors. (For an overview of the different results the reader is advised to consult the survey paper [4].) Disjoint translates of a convex compact set soon became a favorite field of research. For the special case when the domains are disjoint congruent circles Danzer [2] proved

Theorem D. Every finite T(5)-family of disjoint congruent discs in the plane has property $T$.

* This research was partially supported by the Hungarian Science Foundation OTKA, Grant Nos. T037752 and T038397. 
$\mathrm{He}$ also points out that if the disjointness hypothesis or the assumption that the discs are congruent is dropped, then the claim of Theorem D does not hold anymore even if property $T(5)$ is replaced by property $T(r)$ for any fixed $r$.

Grünbaum [5] conjectured, however, that property $T(5)$ implies the existence of a transversal if the family consists of disjoint translates of a compact convex set.

It was Tverberg [11] who finally verified this famous old conjecture proving:

Theorem T. Every finite T(5)-family of disjoint translates of a compact convex domain in the plane has property $T$.

Although the statement of Theorem $\mathrm{T}$ does not hold anymore if property $T(5)$ is relaxed to property $T(4)$, Katchalski and Lewis found [10] that even property $T(3)$ implies the existence of a line which is "almost" a transversal to the family in the following sense:

Theorem KL. There exists a universal integer constant g such that to every finite T(3)family of disjoint translates of a compact convex domain, a line can be found intersecting all but $g$ members of the family.

Katchalski and Lewis expect $g$ to be a small number. Constructions given for discs by Bezdek [1] show that in general $g$ cannot be smaller than 2. Holmsen [6]-[8] proved that $g \leq 22$ and found that $g$ is 4 if the domains are translated copies of a parallelogram. In the case of discs, $g \leq 12$ is the best known result, due to Kaiser [9].

In the present paper the term "almost" is used in a different sense. Instead of counting the exceptional translates which the best line fails to meet we suggest a quatitative approach and measure the failure by the maximal distance of the best line from the translates, or by the double of this: i.e. by the width of the narrowest strip intersecting all members.

The transversal strip of a family of translates is a closed (parallel) strip intersecting all members of the family and the width of the narrowest transversal strip is the transversal width of the family.

Using this term the special case of an often cited much more general result of Eckhoff [3] can be reformulated in the following way:

Theorem E. To every finite T(3)-family of disjoint discs of diameter 1 there exists a transversal strip of width 1 .

Our main goal in this paper is to improve this bound by establishing the following:

Theorem. To every finite T(3)-family of disjoint discs of diameter 1 there exists a transversal strip of width $<0.65$.

Eckhoff expects that the minimal value $w_{5}$ is attained for a regular arrangement of five discs (Fig. 1). This expectation can be expressed in the following:

Conjecture. To every finite T(3)-family of disjoint discs of diameter 1 there exists a transversal strip of width $\leq w_{5}=2 \sin (\pi / 10)=0.618 \ldots$ 


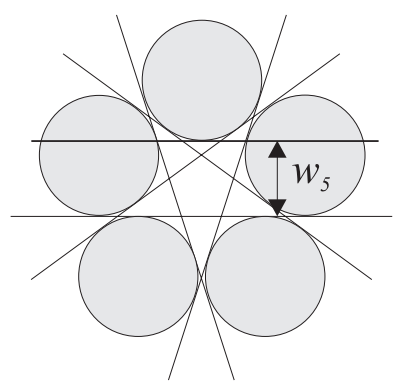

Fig. 1

Notice that the configuration displayed in Fig. 1 is of property $T(4)$, i.e. the truth of this conjecture would imply that even property $T(4)$ cannot guarantee a narrower transversal strip than property $T(3)$ does.

Sets $S_{1}$ and $S_{2}$ are said to be separated if a straight line $\lambda$ disjoint from $S_{1}$ and $S_{2}$, exists such that every line segment connecting a point of $S_{1}$ with a point of $S_{2}$ is intersecting $\lambda$. We say that $S_{1}$ and $S_{2}$ are widely separated if a strip $\sigma$ of unit width, disjoint from $S_{1}$ and $S_{2}$, exists such that every line segment connecting a point of $S_{1}$ with a point of $S_{2}$ is intersecting $\sigma$.

The union of all straight lines intersecting two disjoint discs $D(X)$ and $D(Y)$ is a simply connected closed unbounded domain, the boundary of which consists of parts of the four lines tangent to both discs (Fig. 2(a)). This unbounded domain is called the sheaf belonging to the two discs and is denoted by $\Sigma(X, Y)$. Clearly, a third disc has a common transversal with $D(X)$ and $D(Y)$ if and only if it intersects the sheaf $\Sigma(X, Y)$.

The outer parallel domain of radius $\frac{1}{2}$ of sheaf $\Sigma(X, Y)$, which is the locus of the centers of the discs intersecting $\Sigma(X, Y)$, is called the center sheaf belonging to $X$ and $Y$ and is denoted by $\Sigma^{\mathrm{c}}(X, Y)$. Two of the (at most) six lines generating the boundary of the center sheaf $\Sigma^{\mathrm{c}}(X, Y)$ are non-separating tangent lines to both unit discs $U(X)$ and $U(Y)$ and the four other lines are tangent to one of the two unit discs and pass through the center of the other one (see Fig. 2(b)).

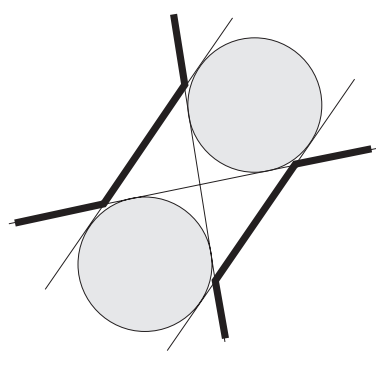

(a)

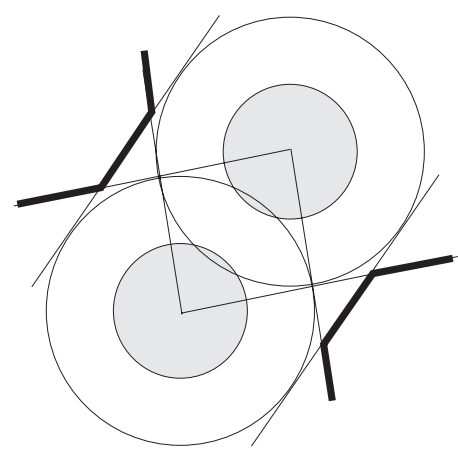

(b)

Fig. 2 
The generalized center sheaf belonging to two orthogonal segments also play an important role in the proof. This is the union of all center sheaves belonging to a pair of discs, one of them centered in the first segment and the other centered in the second one. The generalized center sheaf is a domain bounded by parts of at most six straight lines-like in the case of the common center sheaf, however, the generalized center sheaf is usually not symmetric. $\Sigma^{\mathrm{c}}\left(\overline{X_{1} X_{2}}, \overline{Y_{1} Y_{2}}\right)$ denotes the generalized center sheaf belonging to the segments $X \in \overline{X_{1} X_{2}}$ and $Y \in \overline{Y_{1} Y_{2}}$, where $\overline{X_{1} X_{2}}$ and $\overline{Y_{1} Y_{2}}$ denote the segments on the $x$ - and $y$-axis connecting $X_{1}$ and $X_{2}$ and $Y_{1}$ and $Y_{2}$, respectively. The notation $\lambda(X Y)$ is used for the line connecting the points $X$ and $Y \cdot \lambda^{\ell}(X Y)$ and $\lambda^{\mathrm{r}}(X Y)$ denote the common non-horizontal tangent lines of sets $X$ and $Y$ on the left and on the right, respectively.

\section{The Proof}

1. Contrary to our claim suppose that a counterexample exists. Following Tverberg's reduction method [11] it can be assumed that the centers are in general position, i.e. no three centers form the vertices of a right-angled triangle.

Tacitly the following well-known fact will also be used:

Lemma. The disjoint sets $S_{1}, S_{2}$ and $S_{3}$ have no common transversal if and only if each can be separated from the union of the other two sets.

Let $\sigma^{\mathrm{c}}$ denote the narrowest strip covering all centers of the family. Assume that $\sigma^{\mathrm{c}}$ is horizontal, and its upper boundary line is the $x$-axis. Then we can assume that there exists a center $A(0,-a)$ on the lower boundary line $y=-a$ of $\sigma^{\mathrm{c}}$ and two centers $B(b, 0)$ and $C(-c, 0)$ on the upper one (the $x$-axis) such that $1.65 \leq a \leq 2$ and $0<b \leq c$. We are going to show that these assumptions lead to contradiction: there exist three discs in the family without a line transversal.

The sketch of the proof is the following. It is a direct consequence of the $T$ (3)-property of the discs about $A, B$ and $C$ that to any given value of $a$ there belongs a feasibility interval for $b$ and for any given $a$ and $b$ a feasibility interval for $c$. As center $C$ must be in $\Sigma^{\mathrm{c}}(A, B)$ our assumption $b \leq c$ implies

$$
b \in\left(0, \frac{1}{\sqrt{4-1 / a^{2}}}\right] .
$$

Since the upper end of the interval is a decreasing function of $a$ and $1 / \sqrt{4-1 / 1.65^{2}}=$ $0,5246 \ldots$ the feasibility interval of $b$ is a subinterval of the interval $[0,0.525]$.

In our proof every pair of values $a \in[1.65,2]$ and $b \in[0,0.525]$ will be considered although not all of these combinations are feasible in a $T(3)$-family. Technically, the intervals will be cut into subintervals: $a \in[1.65,2]$ into five subintervals:

$$
a \in[1.65,1.67],[1.67,1.70],[1.70,1.75],[1.75,1.8],[1.8,2],
$$

and, independently, $b \in[0,0.525]$ into six subintervals:

$$
b \in(0,0.1],[0.1,0.3],[0.3,0.42],[0.42,0.48],[0.48,0.51],[0.51,0.525] .
$$




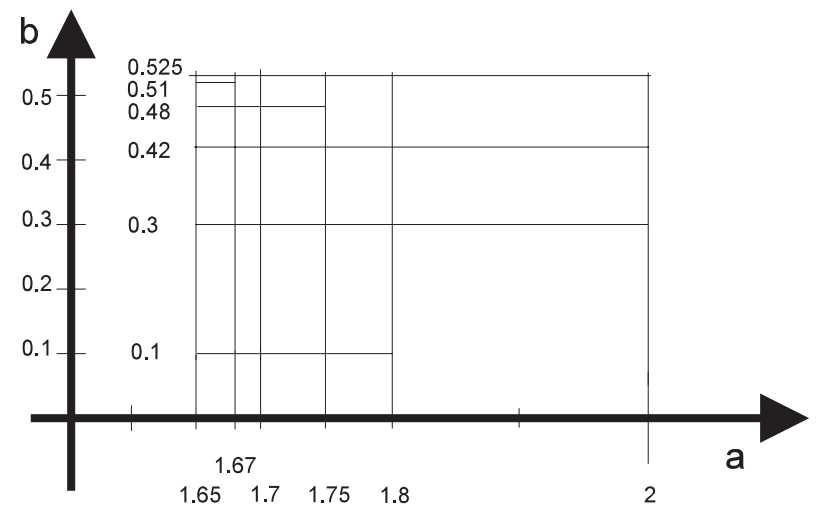

Fig. 3. The "parameter boxes."

This gives 30 "parameter boxes". For some $a$-intervals several $b$-intervals have been united to reduce the calculations. Finally, we have 23 parameter boxes. (For a graphical overview see Fig. 3.)

It will be shown that none of these parameter boxes contains a feasible solution, i.e. a counterexample to the claim of the theorem. Except for the numerical calculations the proof follows the same line for each box therefore it will be carried out for an arbitrary parameter box.

The values $a_{1}<a_{2}$ and $b_{1}<b_{2}$ denote the limits of the selected parameter intervals and $A_{i}\left(0,-a_{i}\right)$ and $B_{i}\left(0, b_{i}\right), i=1,2$, denote the endpoints of the assigned intervals.

2. It is easily seen that in our cases, when the inequalities

$$
1.6 \leq a \leq 2, \quad 0 \leq b_{1}<1, \quad 0.5 \leq c_{1}<1
$$

hold, the generalized center sheaf $\Sigma^{\mathrm{c}}\left(\overline{B_{1} B_{2}}, \overline{A_{1} A_{2}}\right)$ defined by the segments $\overline{A_{1} A_{2}}$ and $\overline{B_{1} B_{2}}$ is lying between two polygonal lines (see Fig. 4), one-on the left—consisting of parts of lines

$$
\lambda^{\ell}\left(U\left(B_{1}\right) A_{1}\right), \lambda^{\ell}\left(U\left(B_{1}\right) U\left(A_{1}\right)\right), \lambda^{\ell}\left(B_{2} U\left(A_{1}\right)\right),
$$

and the other boundary—on the right—consisting of parts of lines

$$
\lambda^{\mathrm{r}}\left(U\left(B_{2}\right) A_{1}\right), \lambda^{\mathrm{r}}\left(U\left(B_{2}\right) U\left(A_{2}\right), \lambda^{\mathrm{r}}\left(B_{1} U\left(A_{1}\right)\right) .\right.
$$

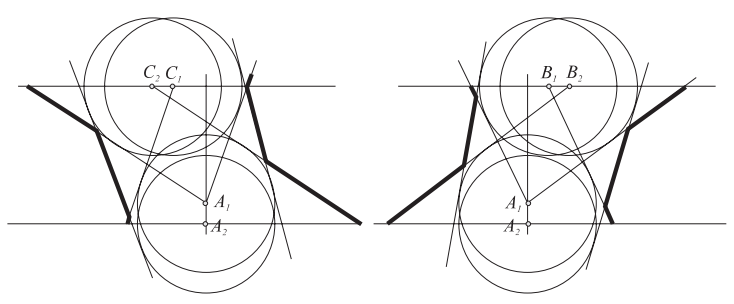

Fig. 4. Generalized center sheaves. 
Analogously, the boundary of $\Sigma^{\mathrm{c}}\left(\overline{C_{1} C_{2}}, \overline{A_{1} A_{2}}\right)$ consists of parts of lines

$$
\lambda^{\ell}\left(U\left(C_{2}\right) A_{1}\right), \lambda^{\ell}\left(U\left(C_{2}\right) U\left(A_{2}\right)\right), \lambda^{\ell}\left(C_{1} U\left(A_{1}\right)\right),
$$

and parts of lines

$$
\lambda^{\mathrm{r}}\left(U\left(C_{1}\right) A_{1}\right), \lambda^{\mathrm{r}}\left(U\left(C_{1}\right) U\left(A_{1}\right)\right), \lambda^{\mathrm{r}}\left(C_{2} U\left(A_{1}\right)\right) .
$$

3. To every box of $a_{1} \leq a \leq a_{2}$ and $b_{1} \leq b \leq b_{2}$ a feasibility interval $\left[-c_{2},-c_{1}\right]$ can be calculated for the Cartesian $x$-coordinate of $C(-c, 0)$ based on the condition $b \leq c$, on the disjointness hypothesis and on the $T$ (3) property of the family. Since the lower bound for $c$ is a decreasing function of $b$ and the upper bound is a decreasing function of both $a$ and $b$ we have

$$
c \geq c_{1}=\max \left(1-b_{2}, 0.5\right)
$$

and, from $\Sigma^{\mathrm{c}}\left(\overline{A_{1} A_{2}}, \overline{B_{1} B_{2}}\right)$,

$$
c \leq c_{2}=\frac{a_{1} \sqrt{\left.a_{1}^{2}+b_{1}^{2}-1\right)}-a_{1}^{2} b_{1}}{a_{1}^{2}-1} .
$$

$C_{i}\left(0,-c_{i}\right), i=1,2$, denote the endpoints of the feasibility segment for $C$.

4. By the $T(3)$ property of the family the three centers $A, B$ and $C$ can always be covered with a strip of width 1 , thus the family, contradicting our claim, contains further discs. All further centers must lie in the bounded polygonal domain which is the intersection of strip $\sigma^{\mathrm{c}}$, center sheaf $\Sigma^{\mathrm{c}}(A, B)$ defined by the discs about $A$ and $B$ and center sheaf $\Sigma^{\mathrm{c}}(A, C)$ defined by the discs about $A$ and $C$ (Fig. 5(a)), moreover, the centers must lie, by the disjointness condition, outside of the unit discs $U(A), U(B)$ and $U(C)$ (Fig. 5(b)).

5. Let $X(A B)$ and $X(A C)$ be the images of the $x$-axis received by reflection on the line $\lambda(A B)$ and $\lambda(A C)$, and let $X^{*}(A B)$ and $X^{*}(A C)$ be the lines through $A$ parallel to $X(A B)$ and $X(A C)$, respectively. Let $\sigma(A B)(\sigma(A C))$ denote the strip bounded by $X(A B)$ and $X^{*}(A B)$ (by $X(A C)$ and $X^{*}(A C)$ ). Strips $\sigma^{\mathrm{c}}, \sigma(A B)$ and $\sigma(A C)$ have, of course, the same width (Fig. 6).

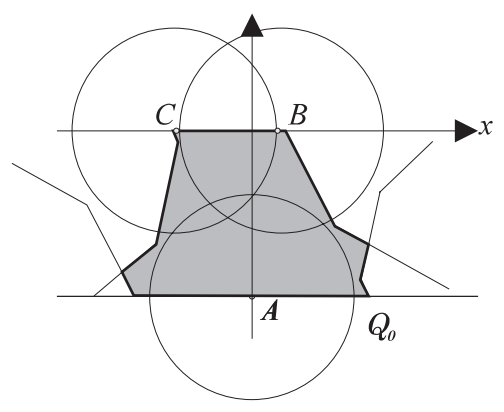

(a)

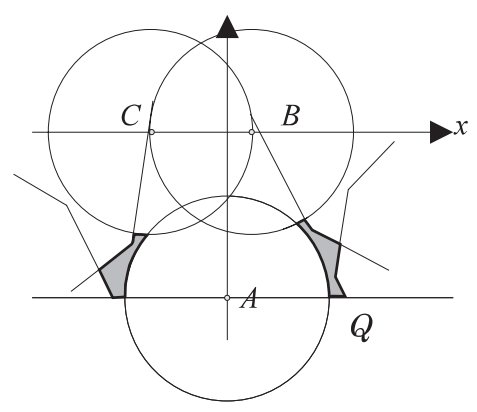

(b)

Fig. 5 


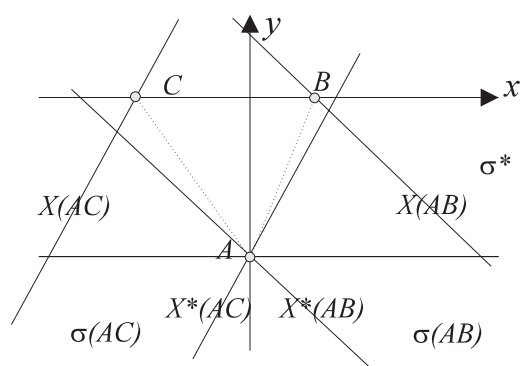

Fig. 6

Since there is no center below the lower boundary line $y=-a$ of the narrowest transversal strip $\sigma^{\mathrm{c}}$ the following holds:

Proposition 1. All centers in the open half-plane $x>0$ are strictly above $X^{*}(A B)$ and all centers in the open half-plane $x<0$ are strictly above $X^{*}(A C)$ for any $a \in[1.65,2]$ and $b \in[0,0.525]$.

The proof is left to the reader. (Remember: no triple of centers is supposed to form a right-angled triangle, hence line $X^{*}(A B)$ is strictly descending and line $X^{*}(A C)$ is strictly ascending.)

Proposition 2. Center $C$ is an inner point of strip $\sigma(A B)$ and center $B$ is an inner point of strip $\sigma(A C)$ for any $a \in[1.65,2]$ and $b \in[0,0.525]$.

The proof is left to the reader. (Hint: consider the intervals $b \leq 0.2$ and $b \geq 0.2$ separately.)

6. If centers $A, B$ and $C$ are allowed to vary in segments $\overline{A_{1} A_{2}}, \overline{B_{1} B_{2}}$ and $\overline{C_{1} C_{2}}$, respectively, then the role of the center sheaves is taken over by the suitable generalized center sheaves. Exploiting the condition that any other center is disjoint from $U(A)$, $U(B)$ and $U(C)$ for any choice in the parameter box, a further reduction of this polygonal domain

$$
Q_{0}=\sigma^{\mathrm{c}} \cap \Sigma^{\mathrm{c}}\left(\overline{A_{1} A_{2}}, \overline{B_{1} B_{2}}\right) \cap \Sigma^{\mathrm{c}}\left(\overline{A_{1} A_{2}}, \overline{C_{1} C_{2}}\right)
$$

is possible. Those points which lie outside of $U(A)$ for at least one $a \in\left[a_{1}, a_{2}\right]$ are the points of the complement of the set of inner points of $U(A)$ for all $a \in\left[a_{1}, a_{2}\right]$, which is the intersection $U\left(A_{1}\right) \cap U\left(A_{2}\right)$. Similarly, a point of $Q_{0}$ is not feasible if it is not outside of $U(B)$ for at least one value of $b \in\left[b_{1}, b_{2}\right]$ and outside of $U(C)$ for at least one value of $c \in\left[c_{1}, c_{2}\right]$. Thus the feasibility domain of further centers reduces-in each box-to

$$
Q=Q_{0} \backslash\left(\left(U\left(A_{1}\right) \cap U\left(A_{2}\right)\right) \cup\left(U\left(B_{1}\right) \cap U\left(B_{2}\right)\right) \cup\left(U\left(C_{1}\right) \cap U\left(C_{2}\right)\right)\right) .
$$




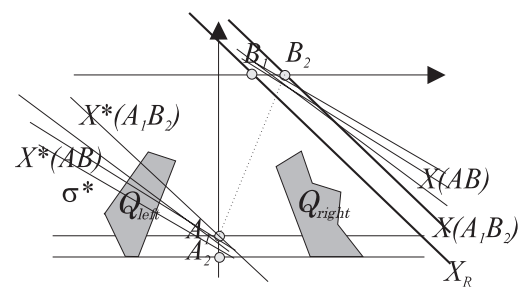

(a)

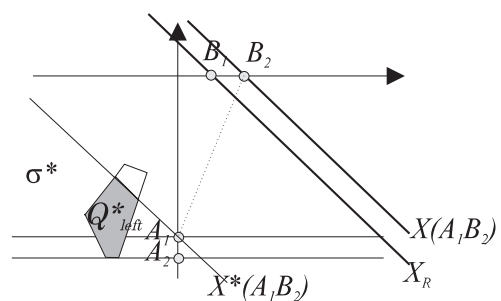

(b)

Fig. 7

(Lying in $Q$ is, of course only a necessary condition to be a potential center and it is far from sufficient.) $Q_{\text {left }}$ and $Q_{\text {right }}$ denote the parts of set $Q$ in the half-planes $x \leq 0$ and $x \geq 0$, respectively.

In the rest of the propositions it is assumed-without mentioning it repeatedly-that the parameters $a$ and $b$ belong to one of the 23 parameter boxes defined above.

Let $X_{\mathrm{R}}$ be the line parallel to $X\left(A_{1} B_{2}\right)$ and passing through $B_{1}$ (Fig. 7(a)) and let $X_{\mathrm{L}}$ be the line parallel to $X\left(A_{1} C_{2}\right)$ and passing through $C_{1}$. Then the following holds:

Proposition 3. Inside the quarter plane $x>0, y<0$, line $X(A B)$ (the upper boundary of $\sigma(A B)$ ) runs above $X_{\mathrm{R}}$ for any $A$ and $B$ of the box and inside the quarter plane $x<0, y<0$ line $X(A C)$ (the upper boundary of $\sigma(A C)$ ) runs above $X_{\mathrm{L}}$ for any $A$ and $C$ of the box.

Proof. On one hand the steepness of line $X(A B)$ is a decreasing function of the steepness of segment $\overline{A B}$, and (within a parameter box) $X(A B)$ is never steeper than $X\left(A_{1} B_{2}\right)$. On the other hand $X(A B)$ intersects segment $\overline{B_{1} B_{2}}$.

We also have

Proposition 4. Domain $Q_{\text {right }}$ lies strictly below $X_{\mathrm{R}}(F i g .7(a))$ and $Q_{\text {left }}$ lies strictly below $X_{\mathrm{L}}$.

Proof. This is easily verified by checking that every vertex of $\operatorname{conv}\left(Q_{\text {right }}\right)$, a polygon, is strictly below $X_{\mathrm{R}}$ and every vertex of $\operatorname{conv}\left(Q_{\text {left }}\right)$ is strictly below $X_{\mathrm{L}}$.

Then the feasibility domain $Q_{\text {right }}$ has no point above $X(A B)$ and $Q_{\text {left }}$ has no point above $X(A C)$ for any choice of parameters in the parameter box.

Combining Propositions 1, 3 and 4 we have

Proposition 5. For any choice of parameters in a given box, all centers of the $x>0$ half-plane lie in the interior of $\sigma(A B)$ except for $B$, which is on its boundary and all centers of the $x<0$ half-plane lie in the interior of $\sigma(A C)$ except for $C$, which is on its boundary. 
By Propositions 5 and 2 the set of centers $A, B$ and $C$ and those finite number of further centers which might be in the interior of $\sigma(A B)$ can be covered by a strip narrower than $\sigma^{\mathrm{c}}$. Consequently, then we have

Corollary E. There exists a center $E_{0}$ in the half-plane $x \leq 0$, i.e. in domain $Q_{\text {left }}$, which is not an inner point of strip $\sigma(A B)$.

As the lower boundary $X^{*}(A B)$ of strip $\sigma(A B)$, intersecting segment $\overline{A_{1} A_{2}}$, is never steeper than line $X^{*}\left(A_{1} B_{2}\right)$ the following holds:

Proposition 6. Line $X^{*}(A B)$ either coincides with line $X^{*}\left(A_{1} B_{2}\right)$ or runs below it in the $x<0, y<0$ quarter plane for any choice of parameters of the box.

Hence center $E_{0}$ must lie below $X^{*}\left(A_{1} B_{2}\right)$ or on it. The part of $Q_{\text {left }}$ consisting of points below or on line $X^{*}\left(A_{1} B_{2}\right)$ will be denoted by $Q_{\text {left }}^{*}$ (Fig. 7(b)).

By a similar argument we have

Corollary F. There exists a center $F_{0}$ in the half-plane $x \geq 0$, i.e. in domain $Q_{\text {right }}$, which is not an inner point of strip $\sigma(A C)$.

In what follows $Q_{\text {right }}^{*}$ denotes the domain which is the rest of $Q_{\text {right }}$ having the part above line $X^{*}\left(A_{1} C_{2}\right)$ removed.

7. Some special points play a key role in the rest of the proof. Points $E_{i}\left(x_{E_{i}}, y_{E_{i}}\right), i=$ $1, \ldots 7$, defined below, are selected so that the convex hull of them is covering $Q_{\text {left }}^{*}$ in each case. This simplifies the required calculations.

The first three are points of line $X^{*}\left(A_{1} B_{2}\right) . E_{1}$ is on the upper half of the boundary of disc $U\left(A_{2}\right), E_{2}$ is on line $\lambda^{\ell}\left(U\left(B_{1}\right) U\left(A_{1}\right)\right)$ and $E_{3}$ is on line $\lambda^{\ell}\left(B_{2} U\left(A_{1}\right)\right)$. In some cases-like, e.g. in case of box $a \in[-1.8,-1.75], b \in[0.42,0.525]-E_{2}$ is a vertex and $E_{3}$ is not, while in other cases-like, e.g. in box $a \in[-1.75,-1.7], b \in[0,0.1]-E_{3}$ is a vertex and $E_{2}$ is not (see Fig. 8). $E_{4}$ is the point of intersection of lines $\lambda^{\ell}\left(B_{2} U\left(A_{1}\right)\right)$

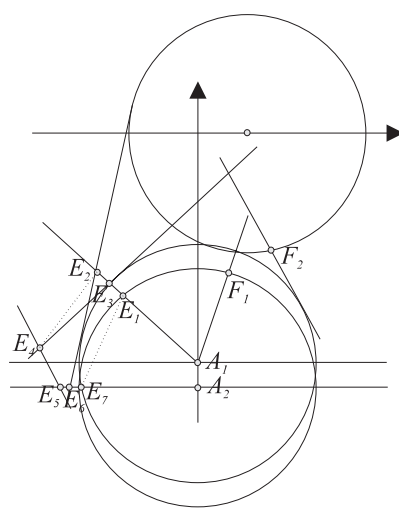

(a)

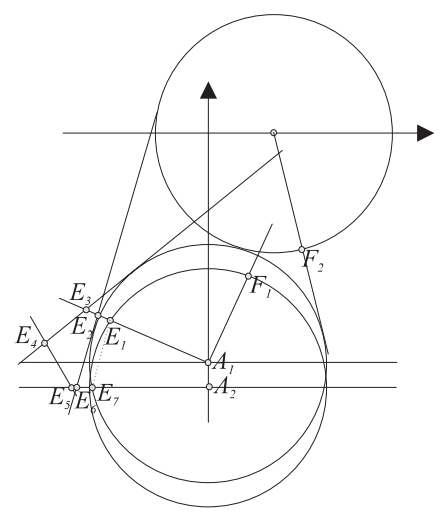

(b)

Fig. 8 


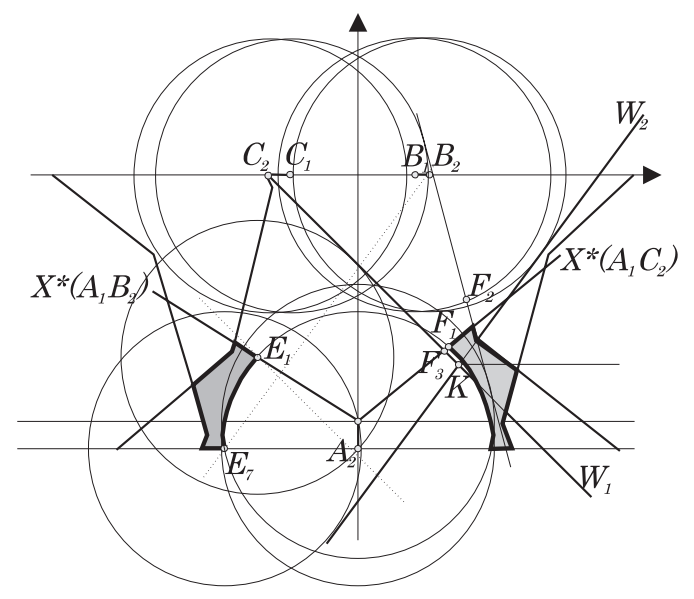

Fig. 9

and $\lambda^{\ell}\left(C_{2} U\left(A_{2}\right)\right) . E_{5}, E_{6}$ and $E_{7}$ are points of line $y=-a_{2}$ on one hand; and lying on $\lambda^{\ell}\left(U\left(C_{2}\right) U\left(A_{2}\right)\right)$, on $\lambda^{1}\left(U\left(B_{1}\right) U\left(A_{1}\right)\right)$ and on the $x<0$ half of the boundary of the disc $U\left(A_{1}\right)$ on the other hand. (We mentioned that the convex hull of $Q_{\text {left }}^{*}$ is always a pentagon. That one of $E_{2}$ and $E_{3}$ which has the smaller $x$-coordinate and $E_{1}$ are vertices of the convex hull of $Q_{\text {left }}^{*}$. That one of $E_{5}$ and $E_{6}$ which has the smaller $x$-coordinate and $E_{7}$ are also vertices of the convex hull of $Q_{\text {left }}^{*}$.)

In the other half-plane let $F_{1}\left(x_{F_{1}}, y_{F_{1}}\right)$ be the point of intersection of $X^{*}\left(A_{1} C_{2}\right)$ with the upper half of the boundary of $U\left(A_{2}\right)$, and let $F_{2}\left(x_{F_{2}}, y_{F_{2}}\right)$ be the point of intersection of $\lambda^{\mathrm{r}}\left(U\left(C_{1}\right) U\left(A_{1}\right)\right)$ and the lower half of the boundary of unit disc $U\left(B_{1}\right)$.

8. Consider now the upper tangent line $W_{1}$ of unit disc $U\left(E_{1}\right)$ passing through $C_{2}$ and the lower tangent line $W_{2}$ of both unit discs $U\left(E_{7}\right)$ and $U\left(B_{2}\right)$ and denote the point of intersection of $W_{1}$ and $W_{2}$ by $K\left(x_{K}, y_{K}\right)$ (Fig. 9).

Simple substitution based on equations of $X^{*}\left(A_{1} B_{2}\right)$ and $W_{1}$ shows that $W_{1}$ is steeper than $X^{*}\left(A_{1} B_{2}\right)$ (in each box). Hence $E_{1}$ is the point of $Q_{\text {left }}^{*}$ nearest to $W_{1}$, thus $Q_{\text {left }}^{*}$ is widely separated from the points above $W_{1}$. This implies

Proposition 7. If $F_{0} \in Q_{\text {right }}^{*}$ is above $W_{1}$ then $E_{0}$ is widely separated from $C \cup F_{0}$.

Similarly, it is easy to check that $W_{2}$ is a lower support line of the outer parallel domain of radius 1 of $Q_{\text {left }}^{*}$ (the critical point being $E_{7}$ ) hence the following holds:

Proposition 8. If $F_{0} \in Q_{\text {right }}^{*}$ is below $W_{2}$ then $F_{0}$ is widely separated from $B \cup E_{0}$.

Let $F_{3}\left(x_{F_{3}}, y_{F_{3}}\right)$ be the point of intersection of $W_{1}$ and $X^{*}\left(A_{1} C_{2}\right)$. Simple substitutions yield, in each parameter box:

Proposition 9. $K$ is an inner point of $U\left(A_{2}\right)$. 
Proposition 10. $\quad F_{3}$ is an inner point of $U\left(A_{2}\right)$.

By Propositions 9 and 10 the segment of $W_{2}$ between $y=-a_{2}$ and $y=y_{K}$ runs inside $U\left(A_{2}\right)$ and segments $\overline{K F_{3}}$ and $\overline{F_{3} F_{1}}$ run inside $U\left(A_{2}\right)$ as well. In addition, the part of $U\left(A_{2}\right)$ above $y=y_{F_{1}}$ is to the left of $X^{*}\left(A_{1} C_{2}\right)$. Hence no point of $Q_{\text {right }}^{*}$ lies to the left of $W_{1}$ above line $y=y_{K}$ and no point of $Q_{\text {right }}^{*}$ lies to the left of $W_{2}$ below line $y=y_{K}$. Consequently, $F_{0}$ lies either above $W_{1}$ or below $W_{2}$.

Let us now decompose domain $Q_{\text {right }}^{*}$ into two parts by line $y=y_{K} \cdot Q^{\text {upper }}$ and $Q_{\text {lower }}$ denote the closures of the upper and lower parts of $Q_{\text {right }}^{*}$, respectively. Using this notation Proposition 7 implies

Proposition 11. $E_{0}$ is widely separated from $C \cup F_{0}$ if $F_{0} \in Q^{\text {upper }}$.

It is easy to check the truth of the next proposition for each box of parameters:

Proposition 12. $F_{0}$ is widely separated from $E_{0} \cup C$ if $F_{0} \in Q^{\text {upper }}$.

Proof. Consider the line $\lambda\left(C_{1} E_{1}\right)$. The substitutions show (in each box) that corner $E_{7}$ is to the left of the line, consequently $\lambda\left(C_{1} E_{1}\right)$ is the support line to the whole domain $Q_{\text {left }}^{*}$, where $E_{0}$ is supposed to lie. Point $F_{1}$ is on the right of $\lambda\left(C_{1} E_{1}\right)$ and its distance from the line is larger than 1 . As $\lambda\left(C_{1} E_{1}\right)$ is steeper than $X^{*}\left(A_{1} C_{2}\right)$ the line parallel to it and passing through $F_{1}$ is a support line of $F_{1} \cup Q^{\text {upper }}$, thus every point $F_{0}$ of it is widely separated from $E_{0} \cup C$.

We need also

Proposition 13. $C$ is widely separated from $E_{0} \cup F_{0}$ if $F_{0} \in Q^{\text {upper }}$.

Proof. Since the shape of $Q^{\text {upper }}$ depends on the choice of the parameter box, this proposition requires more, however simple, calculation to verify that the distance of $C$ and $Q_{\text {left }}^{*} \cup Q^{\text {upper }}$ is larger than 1 (in each box) as claimed.

Based on Propositions 11-13 we have

Corollary U. The discs about $C, E_{0}$ and $F_{0}$ have no common transversal if $F_{0} \in Q^{\text {upper }}$, thus $F_{0}$ cannot lie in the upper part of $Q_{\text {right }}^{*}$.

Analogously, since $Q_{\text {lower }}$ is below line $W_{2}$, Proposition 8 implies

Proposition 14. $\quad F_{0}$ is widely separated from $E_{0} \cup B$ if $F_{0} \in Q_{\text {lower }}$.

The last two steps are rather simple to prove:

Proposition 15. $E_{0}$ is widely separated from $B \cup F_{0}$ if $F_{0} \in Q_{\text {lower }}$. 
Proof. The strip of unit width between lines $y=-4 x$ and $y=-4 x-\sqrt{17}$ widely separates $E_{1}$ and $K$ while the lines through $E_{1}$ and $K$ and parallel to $y=-4 x$ are support lines of $Q_{\text {left }}^{*}$ and $K \cup Q_{\text {lower }}$, respectively. Hence the claim is proved.

Proposition 16. $B$ is widely separated from $E_{0} \cup F_{0}$ if $F_{0} \in Q_{\text {lower. }}$.

Proof. This is a simple consequence of the fact that the topmost point of $Q_{\mathrm{left}}^{*}\left(E_{2}\right.$ or $E_{3}$ ) as well as point $K$ lie below line $y=-1$.

Propositions 14-16 imply

Corollary L. The discs about $B, E_{0}$ and $F_{0}$ have no common transversal if $F_{0} \in Q_{\text {lower, }}$, thus $F_{0}$ cannot lie in the lower part of $Q_{\text {right }}^{*}$.

Corollaries E, F, U and L together yield contradiction and this concludes the proof to the theorem.

\section{Acknowledgement}

The author is indebted to the unknown referee for enhancing the readability of the paper.

\section{References}

1. A. Bezdek, On the transversal-conjecture of Katchalski and Lewis, in Intuitive Geometry, Szeged (Hungary), Colloquia Mathematica Societati János Bolyai, vol. 63, North-Holland, Amsterdam, 1991, pp. 23-25.

2. L. Danzer, Über ein Problem aus der kombinatorischen Geometrie, Arch. Mat. 8 (1957), 347-351.

3. J. Eckhoff, Transversalenprobleme in der Ebene, Arch. Math. 24 (1973), 191-202.

4. J. E. Goodman, R. Pollack and R. Wenger, Geometric transversal theory, in New Trends in Discrete and Computational Geometry (ed. J. Pach), Algorithms and Combinatorics, 10, Springer-Verlag, Berlin, 1993, pp. 163-198.

5. B. Grünbaum, On common transversals, Arch. Math. (Basel) 9 (1958), 465-469.

6. A. Holmsen, Geometric transversals for families of disjoint translates in the plane, Technical Report, Department of Pure Mathematics, University of Bergen, 2000.

7. A. Holmsen, New bounds on the Katchalski-Lewis transversal problem, Discrete Comput. Geom. 29 (2003), 395-408.

8. A. Holmsen, A transversal theorem in the plane, Unpublished manuscript.

9. T. Kaiser, Line transversals to unit discs, Discrete Comput. Geom. 28 (2002), 379-387.

10. M. Katchalski and T. Lewis, Cutting families of convex sets, Proc. Amer. Math. Soc. 79 (1980), 457-461.

11. H. Tverberg, Proof of Grünbaum's conjecture on common transversals for translates, Discrete Comput. Geom. 4 (1989), 191-203.

Received January 7, 2004, and in revised form August 2004. Online publication July 8, 2005. 